\title{
COMPORTAMIENTO EN CAUTIVERIO DEL MORROCOY (Geochelone carbonaria) DURANTE LA ÉPOCA REPRODUCTIVA
}

\section{CAPTIVITY BEHAVIOR OF REDFOOT TORTOISE (Geochelone carbonaria) DURING THE REPRODUCTIVE SEASON}

\author{
MARTÍNEZ-TORRES, VÍCTOR A ${ }^{1}$ Zootec. GÓMEZ-ALDANA, LUIS E ${ }^{1}$ Zootec. \\ DE LA OSSA, V. JAIME ${ }^{1^{*}}$ Doctor. \\ ${ }^{1}$ Facultad de Ciencias Agropecuarias, Universidad de Sucre, Colombia. ${ }^{2}$ \\ Profesor Titular Universidad de Sucre, Facultad de Ciencias Agropecuarias, \\ Colombia. Grupo de Investigación en Biodiversidad Tropical. \\ *Correspondencia: jaimedelaossa@yahoo.com
}

Recibido: 30-11-2009; Aceptado: 13-02-2010

\section{Resumen}

Con fin de conocer el comportamiento reproductivo de G. carbonaria en cautiverio, se seleccionaron doce ejemplares, tres machos y nueve hembras, de tamaños similares, con una talla promedio de $34,35 \mathrm{~cm}$ de longitud total de caparazón ( $D S=4,74)$. Los especímenes seleccionados fueron mantenidos en cautiverio (densidad de 0,08 ind $/ \mathrm{m}^{2}$ ). El comportamiento reproductivo se evaluó entre los meses de febrero y abril del año 2009. Durante el mes de febrero no se observó ningún despliegue de tipo reproductivo, sin embargo éste aumentó conforme se incrementaban las lluvias. En las categorías y eventos evaluados se encontraron diferencias estadísticamente significativas en los eventos vocalización $(p<0,001)$ y cópula $(p<0,001)$. El periodo dedicado a la evaluación del comportamiento reproductivo, sugiere que $G$. carbonaria en cautiverio lleva a cabo dicho comportamiento en las horas de la tarde. No se observó diferencia estadísticamente significativa $(p>0,05)$ con respecto al tiempo dedicado a los eventos de las categorías de manutención, locomoción, alimentación, social agonístico y social no agonístico. Las observaciones registradas señalan que $G$. carbonaria en cautiverio no presenta limitaciones reproductivas.

Palabras clave: Geochelone carbonaria, comportamiento reproductivo, cautiverio, Sucre, Colombia.

\section{Abstract}

With the purpose of know the reproductive behavior of $G$. carbonaria in captivity, 12 individuals: three males and nine females were selected, of similar sizes, with an average of $34.35 \mathrm{~cm}$ of total length of shell (DS=4.74). The selected especímenes was 
maintained in captivity (density $=0.08 \mathrm{ind} / \mathrm{m}^{2}$ ). The reproductive behavior was evaluated during February to April of 2009. During February unfolding of reproductive type was not observed, however this increased conforms to the rains. In the categories and evaluated events differences statistically significant were observed in the vocalization events $(p<0.001)$ and copulation $(p<0.001)$. The period dedicated to the evaluation of the reproductive behavior, suggests that $G$. carbonaria in captivity carry out this behavior in the afternoon. Statistical significant differences were not observed $(p>0.05)$ with respect to the time dedicated to the events of the maintenance categories, locomotion, feeding, social agonistic and social non agonistic. The registered observations point out that $G$. carbonaria in captivity not presents reproductive limitations.

Key words: Geochelone carbonaria, reproductive behavior, captivity, Sucre, Colombia.

\section{Introducción}

El género Geochelone (Chelonoidis) en Colombia incluye las especies $G$. carbonaria y $G$. denticulada. La primera se encuentra distribuida en la cuenca del Amazonas, en el área comprendida entre Chocó, Norte de Santander, Orinoquía, inclusive en las islas de Providencia, mientras que ésta ultima espacie se encuentra en los Llanos Orientales, Vaupés y Amazonas (MEDEM, 1962). G. carbonaria habita ampliamente al oriente de los Andes, Colombia, Venezuela, Brasil, Guayanas, Bolivia, Paraguay y Argentina; se han reportado colonias en las islas de San Adres y Providencias y las Antillas y sur oriente de Panamá (RUEDA et al., 2007). En Colombia, actualmente G. carbonaria y G. denticulata están catalogadas como "amenazadas" según la UICN, y están e incluidas en el apéndice II del CITES (RUEDA, 2006).

G. carbonaria prefiere bosques secos, sabanas, montes de galería y moriches, donde $G$. denticulata está ausente; con frecuencia se halla en selvas tropicales húmedas y regiones de pie de monte llanero, lo que sugiere que es una especie adaptada a vivir en regiones de estación seca prolongada, a diferencia de G. denticulata, que prefiere hábitats húmedos (LEGLER, 1963; CASTAÑO y LUGO, 1979).

PÁEZ et al. (2006) afirman que en Colombia alrededor del $56 \%$ de la especies de tortugas presentan problemas de conservación, una de las principales causas es la sobre explotación. RUEDA (2006) incluyó a G. carbonaria entre las poblaciones de vertebrados amenazados de extinción en la cuenca del río Sinú, departamento de Córdoba, y sugiere que se debe resaltar el valor de las especies endémicas y la biota presente en áreas restringidas.

OJASTI (1995) señala que la creciente presión pública en países importadores de mascotas en contra de la captura de ejemplares del medio silvestre, 
favorece la cría en cautiverio, por lo que se debe asesorar y orientar a las iniciativas privadas de cría en cautiverio. Por otra parte, HERNÁNDEZ (1997) argumenta que esta importancia no está acorde con las pocas investigaciones sobre la biología de $G$. carbonaria tanto en cautiverio como en vida silvestre, por lo cual se deben desarrollar estudios que permitan conocer mejor a la especie, para así desarrollar políticas que no pongan en peligro su supervivencia.

A partir de 1990, la cría en cautiverio de G. carbonaria se estableció como una medida conservacionista de la fauna silvestre. Entre los aspectos que se destacan por tener un efecto negativo sobre la conservación se cuentan la agresión involuntaria del hombre, los cambios climatológicos, las condiciones adversas del terreno y la depredación (GONZALES, 1997).

CASTAÑO y MEDEM (2002) plantean que es necesario estudiar las áreas donde $G$. carbonaria subsiste naturalmente, para desarrollar estudios sobre su ecología y biología, con el fin de apoyar la creación de las áreas protegidas, precisando que es necesario tener en cuenta que es una especie que se reproduce muy bien en cautiverio, es la mejor candidata para programas de zoocría aprovechando su importancia para las comunidades, su rusticidad y el conocimiento popular, complementándolo con la investigación científica y programas de educación.

En el presente trabajo se analizan los diferentes comportamientos que de manera grupal presenta $G$. carbonaria, enfatizando en las pautas de comportamiento más representativamente observadas durante la temporada reproductiva, con el fin de dilucidar y ampliar a mediano y largo plazo las posibilidades de manejo ex situ con fines conservacionistas activos.

\section{Materiales y Métodos}

Área de estudio: El estudio se realizó en la granja El Perico, perteneciente a la Universidad de Sucre, ubicada en la margen izquierda de la vía que conduce de Sincelejo a la cabecera municipal de Sampués, distante a $11 \mathrm{~km}$ de la primera localidad mencionada. Con coordenadas geográficas de $9^{\circ} 12^{\prime} \mathrm{N}$ y $75^{\circ}$ 26' O (IGAC, 1969). Dicha sede posee una extensión de 11,8 ha. Se caracteriza por ser una zona perteneciente al bosque seco tropical o dentro del zonobioma tropical alternohigríco (HERNÁNDEZ y SÁNCHEZ, 1992).

Grupo experimental: Para el estudio se utilizó un grupo de doce ejemplares adultos de G. carbonaria (morrocoy), constituido por nueve hembras y tres machos, de tamaños similares, con una talla promedio del grupo de $34,35 \mathrm{~cm}$ 
de longitud total de carapax ( $D S=4,74$ ), alojados en un encierro con un área de $150 \mathrm{~m}^{2}$ (10 m x $15 \mathrm{~m}$; Fig. 1). El encierro contaba con cuatro estanques de cemento de $1 \mathrm{~m}^{2}$, donde se les proporcionaba agua que les permitiera beber y bañarse.

Para registrar la información se utilizaron tablas, cronometro (Timex $®$ ) y binoculares $10 \times 30$ (Leyca $\AA$ ); además se marcaron los animales con tinta blanca biodegradable en la parte dorsal y costal del caparazón con números del cuatro al doce para las hembras y del uno al tres para los machos (DE LA OSSA, 1999).

Observaciones y registros: Las observaciones se realizaron según MARTIN y BATESON (1993), mediante muestreos de barrido, con mediciones continuas de las actividades desarrolladas por uno o varios individuos, durante dos sesiones diarias; entre las 7:00 y las 10:00, y entre las 14:00 y las 17:00 horas, durante tres meses (febrero - abril del 2009), época relacionada directamente con la fase reproductiva.

Se definieron conductas (estados) que se agruparon en categorías (pautas y eventos) comportamentales y se relacionaron con aspectos reproductivos dentro de una conducta adaptando la metodología de MIKICH (1991), a partir de las mediciones se elaboraron diagramas para facilitar la interpretación del cubrimiento de las categorías y conductas detectadas, trazando en una gráfica una línea interpretativa que permitiera diferenciar conductas particulares 0 especificas de tipo individual que por su naturaleza diferenciable se hicieran conspicuas (PORTO y PIRATELLI, 2005).

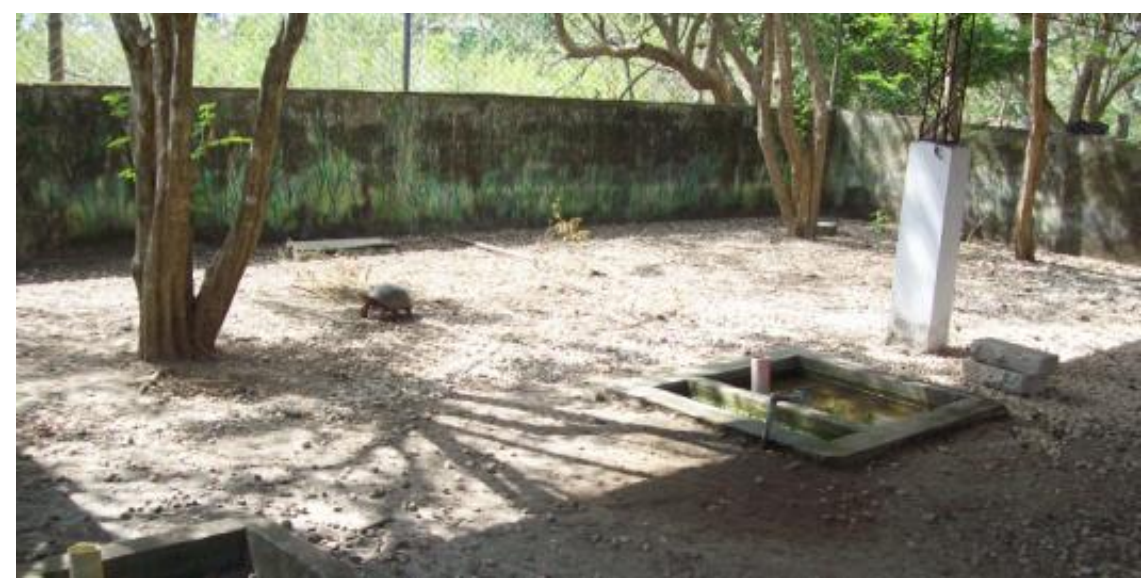

Figura 1. Área de experimentación en la granja El Perico, Universidad de Sucre

Análisis estadístico: Para este estudio se utilizó un diseño al azar. Estas mediciones incluyen número de sucesos y tiempo promedio dedicado a cada evento diariamente. Para determinar si existían diferencias entre las categorías 
y conductas evaluadas, se realizó una prueba de varianza por rangos de Kruskal-Wallis (ZAR, 1996).

\section{Resultados}

Fueron identificadas y descritas 26 categorías (c) de las siguientes conductas (C) evaluadas: manutención $(n=2)$, locomoción $(n=3)$, alimentación $(n=2)$, social no agonística $(n=8)$, socia I agonística $(n=7)$, alerta $(n=2)$, sonora $(n=2)$ (Tabla 1).

\begin{tabular}{|c|c|c|c|c|}
\hline Conducta (C) & $\begin{array}{l}\text { Categorías } \\
\text { (c) }\end{array}$ & $\begin{array}{c}\text { Tiempo } \\
\text { (min) }\end{array}$ & DS & $\begin{array}{l}\text { Actividad } \\
\text { diaria (\%) }\end{array}$ \\
\hline \multirow{2}{*}{ Manutención } & Asoleo & 25 & 12,083 & 1,19 \\
\hline & Refugio & 126,67 & 34,79 & 17,36 \\
\hline \multicolumn{4}{|l|}{ Total actividad diaria (\%) } & 18.55 \\
\hline \multirow[b]{3}{*}{ Locomoción } & Desplazamiento corto & 27,29 & 4,47 & 3,5 \\
\hline & Desplazamiento medio & 30,20 & 5,71 & 3,81 \\
\hline & Desplazamiento largo & 57,04 & 13,95 & 9,33 \\
\hline \multicolumn{4}{|l|}{ Total actividad diaria (\%) } & 16,64 \\
\hline \multirow[t]{2}{*}{ Alimentación } & Comer & 35,42 & 12,51 & 4,30 \\
\hline & Beber & $0,67-1$ & 0 & 0,71 \\
\hline \multicolumn{4}{|l|}{ Total actividad diaria (\%) } & 5,01 \\
\hline \multirow{8}{*}{$\begin{array}{c}\text { Social no agonística (no } \\
\text { agresiva) }\end{array}$} & Agrupado por sexo & 24,92 & 13,16 & 13,87 \\
\hline & Agrupado entre sexo & 14,92 & 6 & 4,7 \\
\hline & Separados por sexo & 26,33 & 8,05 & 17,83 \\
\hline & Separados entre sexo & 14,33 & 8,45 & 5,8 \\
\hline & Movimientos de cabeza por sexo & 9,2 & 1,4 & 0,15 \\
\hline & Movimientos de cabeza entre sexo & 12,2 & 1,42 & 0,21 \\
\hline & Olfateo por sexo & 18,33 & 15,03 & 0,053 \\
\hline & Olfateo entre sexo & 10,83 & 8,01 & 0,45 \\
\hline \multicolumn{4}{|c|}{ Total actividad diaria (\%) } & 43,06 \\
\hline \multirow{7}{*}{$\begin{array}{l}\text { Social agonística } \\
\text { (agresiva) }\end{array}$} & Agresión efectiva entre machos & 3 & 2,7 & 0,016 \\
\hline & Agresión efectiva macho hembra & 2,5 & 1.9 & 0,022 \\
\hline & Agresión efectiva hembra macho & 5 & 0 & 0,0056 \\
\hline & Agresión efectiva entre hembras & 7 & 2,73 & 0,070 \\
\hline & Agresión ritualizada entre machos & 5 & 0 & 0,0056 \\
\hline & Agresión ritualizada hembra macho & 5 & 0 & 0,0056 \\
\hline & Agresión ritualizada macho hembra & 7 & 2,73 & 0,016 \\
\hline \multicolumn{4}{|l|}{ Total actividad diaria (\%) } & 0,14 \\
\hline \multirow[t]{2}{*}{ Alerta } & Observación del intruso & 10,67 & 4,39 & 0,07 \\
\hline & Vigilancia total & 85,33 & 23,91 & 13,43 \\
\hline \multicolumn{4}{|l|}{ Total actividad diaria (\%) } & 14,50 \\
\hline \multirow[t]{2}{*}{ Sonora } & Cópula & 10,41 & 4,5 & 0,41 \\
\hline & Vocalización hacia hembra & 28,33 & 12,12 & 1,69 \\
\hline \multicolumn{4}{|l|}{ Total actividad diaria (\%) } & 2,10 \\
\hline
\end{tabular}




\section{Descripción categórica para la conducta de manutención}

Asoleado: Se desarrolló tanto en las horas de la mañana como de la tarde. Los animales elegían lugares donde se facilitaba esta actividad, exponiendo el carapax, extremidades y cuello al sol, este último pueden extenderlo sobre el suelo o proyectarlo hacia arriba en un ángulo de $45^{\circ}$.

Refugio: utilizaban las esquinas del encierro, troncos de árboles y bebederos sin agua como sitio de resguardo. Se retiraban de los refugios en el tiempo transcurrido entre las 6:40 y 7:30 horas y alrededor de las 16:50 horas iniciaban nuevamente la búsqueda de refugios, los cuales en generalmente eran frecuentado por los mismos animales. En ocasiones algunos individuos permanecían por varias horas e incluso días sin realizar ninguna actividad.

\section{Descripción categórica para la conducta locomoción}

Desplazamientos: desplazamiento corto (menos de $1 \mathrm{~m}$ ), desplazamiento medio (1 a $2 \mathrm{~m}$ ) y desplazamiento largo (más $2 \mathrm{~m}$ ). El desplazamientos estuvo relacionado con la búsqueda de refugio, alimento, lugares para asolearse y búsqueda de hembras por parte de los machos.

\section{Descripción categórica para la conducta alimentación}

Comer: Los animales olfateaban y realizaban pequeños movimientos de cabeza, para analizar cualquier material que potencialmente fuera un recurso alimenticio, el cual acepta o rechaza. El área en el cual se encontraba disponible el alimento era revisada minuciosamente. Los datos registrados indican que la búsqueda de dicho recurso era realizada cada dos días.

Beber: El animal tiende a sumergir alrededor de $1 / 3$ de su cabeza en el espejo de agua, entre 40 y 60 segundos. El consumo de agua por parte de $G$. carbonaria fue registrado cada 4 días.

\section{Descripción categórica para la conducta social no agonística}

Agrupación: En esta categoría se encuentra la agrupación por sexos iguales y agrupación por sexos diferentes. La notable preferencia mostrada hacia las esquinas del encierro y la inexistencia de refugios disponibles favoreció la conformación de grupos de sexos iguales (hembras), los machos mostraron una mayor tendencia a estar aislados en las primeras horas de la mañana y al atardecer, se les observaba haciendo parte de grupos de sexos diferentes al momento de acercarse a inspeccionar a las hembras que se encontraban separadas o agrupadas. 
Separación: Se le hizo un seguimiento a la separación por sexos iguales y separación por sexos diferentes. Ambos se presentaron al dispersarse parte de los individuos que conformaban los grupos de sexos iguales y diferentes, circunstancialmente se percibió una separación por sexos diferentes luego de una serie de olfateos. La separación por sexos iguales se facilitó por la predilección por parte de algunos animales a lugares específicos dentro del encierro.

Movimientos de cabeza: Al igual que la conducta anteriormente descrita, ésta se observó tanto en sexos iguales como diferentes. Los movimientos de cabeza entre sexos iguales al inicio de la investigación (febrero) fueron más marcados con respecto al tiempo empleado en el registro total de la investigación. En hembras se observó dicho comportamiento en encuentros circunstanciales, los cuales duraban un máximo de 3 minutos. El movimiento de cabeza entre machos se evidenció cuando se presentó acercamiento de dos machos hacia una misma hembra. La información recopilada indica que por cada 205,6 encuentros, sólo en uno se presentaron movimientos de cabeza para sexos iguales, mientras que para el caso de encuentros entre sexos diferentes, por cada 49 encuentros sólo en uno se presentaron movimientos de cabeza.

Olfateo: Fue evidente en encuentros fortuitos y persecuciones de machos hacia hembras, éste fue realizado en la parte caudal y costal de la hembra, según la posición, con leves sacudidas de cabeza acompañadas de desplazamientos cortos. Este evento se manifestó entre individuos del mismo sexo como entre individuos de sexo diferente. Según lo percibido, por cada 70,6 encuentros se observó un solo olfateo entre individuos de sexo diferente y 406,57 entre individuos del mismo sexo.

\section{Descripción categórica para la conducta social agonística}

Agresión efectiva entre machos: Se observo en encuentros por disputa de un área o sitio estratégico para la observación, destacándose las mordidas en las extremidades, seguido de golpes fuertes en el carapax del contrincante, el animal introduce su cuello en su coraza y se balancea hacia atrás, y luego se proyecta hacia el contrincante rápidamente propinando de esta manera varios golpes fuertes y certeros.

Agresión efectiva macho hembra: Fue observado durante el mes de febrero en encuentros imprevistos, el macho claramente rechaza a cualquier individuo que se le acerque, incluso hembras que fue el caso observado, al parecer prefieren estar solos, esta agresión se manifestó con pequeños mordiscos 
seguido de persecuciones ocasionales, hasta que la hembra se aleja lo suficientemente.

Agresión efectiva hembra macho: Al igual que los machos, al inicio de la temporada reproductiva (febrero), la hembras suelen agredir a cualquier individuo del sexo opuesto que se le acerque, en ocasiones suelen responder a la agresión de un macho, mordiendo las extremidades cuando en la persecución este la adelanta.

Agresión efectiva entre hembras: Fue percibida durante la formación de los grupos de descanso. En primer lugar se presentaban una serie de movimientos de cabeza entre ellas, dando lugar posteriormente a mordidas en las extremidades, se observaron empujones entre ellas, finalizando con la huida de la hembra agredida.

Agresión ritualizada entre machos: En la medida en que dos machos o más estén cerca de una hembra, y procedan a olfatearla, tendrá lugar una disputa pasiva, caracterizada por movimientos de cabeza, miradas fijas entre sí, ausencia de vocalizaciones y agresión física. Cabe destacar una posición pasiva y en alerta total en cada uno de los individuos implicados en dicho suceso, que finaliza con la retirada de uno de ellos.

Agresión ritualizada hembra macho: Raramente observada, surge como respuesta de rechazo pasivo al acercamiento del macho, la hembra realiza un desplazamiento corto y se ubica en frente del macho, ambos se observan fijamente; el macho exhibe movimientos de cabeza, la hembra mantiene fija mirada en total quietud, luego el macho detiene los movimientos de cabeza y se aleja.

Agresión ritualizada macho hembra: Frente a una actitud negativa de la hembra a la vocalización del macho, éste inicia una serie de suaves mordidas en las extremidades, a lo cual la hembra responde con pequeños movimientos, seguido nuevamente de una serie de vocalizaciones y movimientos de cabeza por parte del macho, todo esto se repite luego de una pequeña pausa. Durante dicho evento la hembra mantiene una vigilancia total sobre el macho.

\section{Descripción categórica para la conducta de alerta}

Observación del intruso: Fue más evidente durante las primeras dos semanas de observación; el macho toma una posición en la cual el rango de visión está en dirección al intruso (con el cuello extendido en un ángulo de $45^{\circ}$ ), permaneciendo así por varios minutos. 
Vigilancia total: esta actitud fue adoptada luego de realizar desplazamientos (corto, medios y largos) con relación al refugio, o grupo en el que se encontraban, algunos ejemplares permanecen en actitud de observación y en total quietud.

\section{Descripción categórica para la conducta sonora}

Vocalización hacia hembras: Las vocalizaciones antes y durante la cópula, están acompañadas de movimientos de cabeza, el sonido emitido varía en la medida en que el macho abre y cierra la boca. Este evento no garantiza una monta exitosa, tampoco una respuesta positiva por parte de la hembra. Es preciso aclarar que el tiempo dedicado a la vocalización es mayor que el de cópula.

Apareamiento: Este evento se complementa con las conductas alerta, locomoción y olfateo. El macho reconoce los lugares por los cuales se movilizan las hembras, generalmente cerca de la zona de refugio, bebederos y bordes del encierro, se ubican allí y esperan en alerta total.

El macho se desplaza suavemente por detrás o a un costado de la hembra y la olfatea, luego inicia la vocalización con una serie de movimientos de cabeza que van hacia la izquierda con pequeñas sacudidas y culminan con un movimiento brusco hacia el lado opuesto, donde continua desarrollando el mismo patrón de comportamiento; posteriormente intenta colocar una de sus extremidades delanteras sobre la parte caudal y por encima del caparazón, formando así un punto de apoyo, facilitando de esta manera el intento de montar a la hembra y permanecer encima de ella y llevar a cabo la cópula. La vocalización perdura durante la cópula manteniendo el patrón de movimientos de cabeza, con el cuello proyectado hacia la cabeza de la hembra. Durante el proceso, la hembra permanece tranquila, en ocasiones realiza pequeños desplazamientos con pequeñas pausas, con el macho a cuesta. El macho a parte de vocalizar y realizar movimientos de cabeza, ejecuta una serie de movimientos, se suspende hacia arriba con ayuda de sus extremidades traseras y deja caer su cuerpo contra el suelo, al tiempo que se encuentra aferrado a la hembra con sus extremidades delanteras, el movimiento es repetitivo durante la cópula, la hembra responde levantando la parte anterior de su cuerpo, con intento de fuga. En algunas ocasiones, se observó durante la cópula al macho intentar agredir físicamente a la hembra en el rostro.

La descripción gráfica del ritmo de actividad registrado durante el presente trabajo, teniendo en cuenta la frecuencia para cada categoría se presenta en la Fig. 2. 


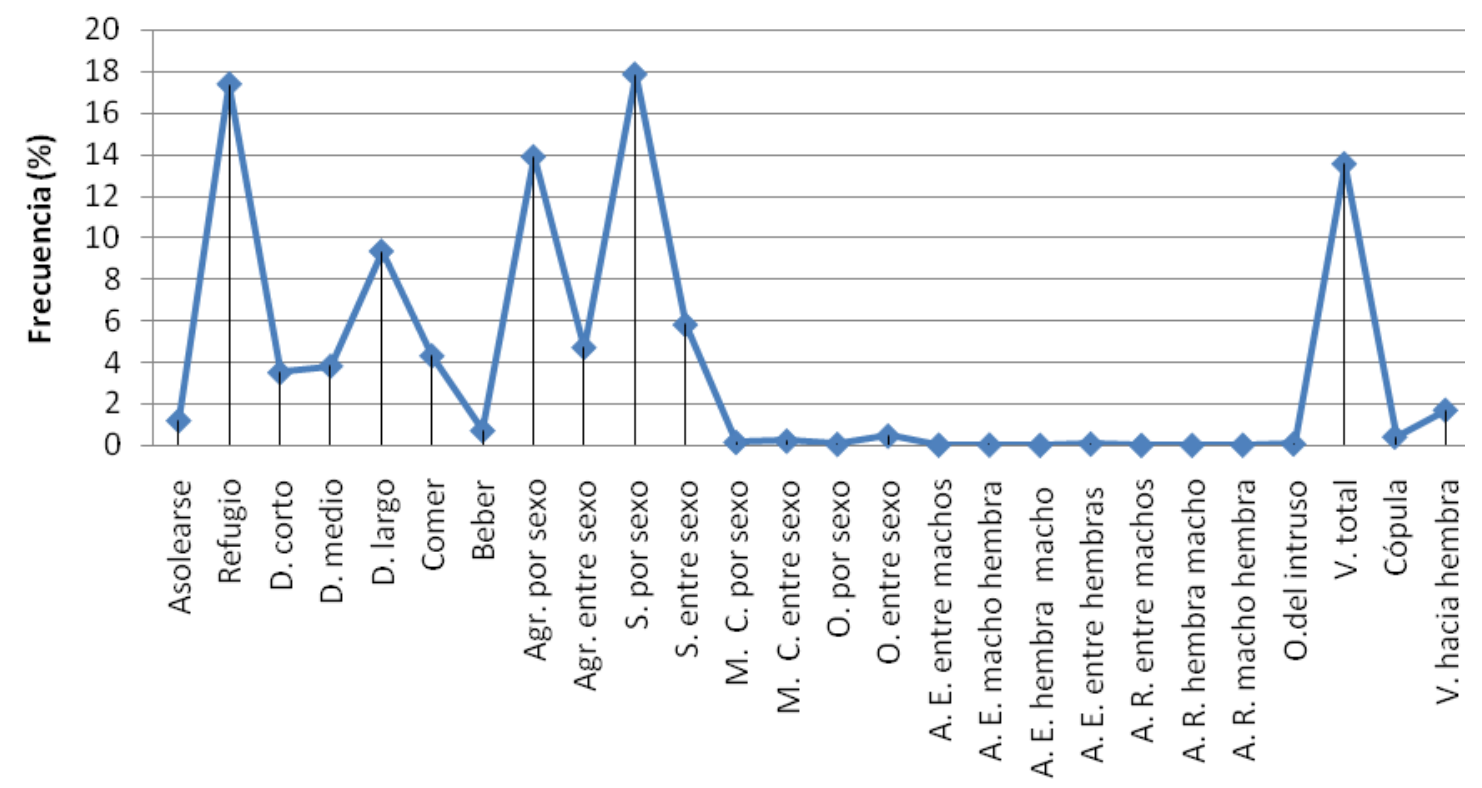

Eventos

Figura 2. Distribución de frecuencia relativa para el tiempo empleado por cada categoría de conducta.

Para comparar el número de sucesos por eventos y el tiempo (minutos) dedicado a cada una de las categorías mediante prueba de Kruskal-Wallis, se obtuvo diferencia significativa con relación al número de sucesos por evento, en las pautas de movimientos de cabeza entre sexo $\left(X_{(1)}^{2}=8,63 ; p<0,001\right)$, olfateo entre sexo $\left(X^{2}{ }_{(1)}=9,42 ; p<0,001\right)$, vocalización $\left(X_{(1)}^{2}=6,04 ; p<0,001\right)$, cópula $\left(X^{2}{ }_{(1)}=9,14 ; p<0,001\right)$ y vigilancia total $\left(X^{2}{ }_{(1)}=6,45 ; p<0,001\right)$ indicando que las medias de los sucesos empleado en cada evento son significativamente diferentes entre sí, confirmando de esta manera que $G$. carbonaria presentan una mayor predisposición a realizar conductas reproductivas y vigilancia total en las horas de la tarde. Al comparar el tiempo empleado por evento en cada uno de las sesiones evaluadas, se encontró diferencias significativas para los actividades de cópula $\left(X^{2}{ }_{(1)}=13,6 ; p<0,001\right)$ y vigilancia total $\left(X_{(1)}^{2}=5,88 ; p<0,001\right)$, revelando que el tiempo dedicado a estas actividades es significativo en las horas de la tarde (Fig. 3). 


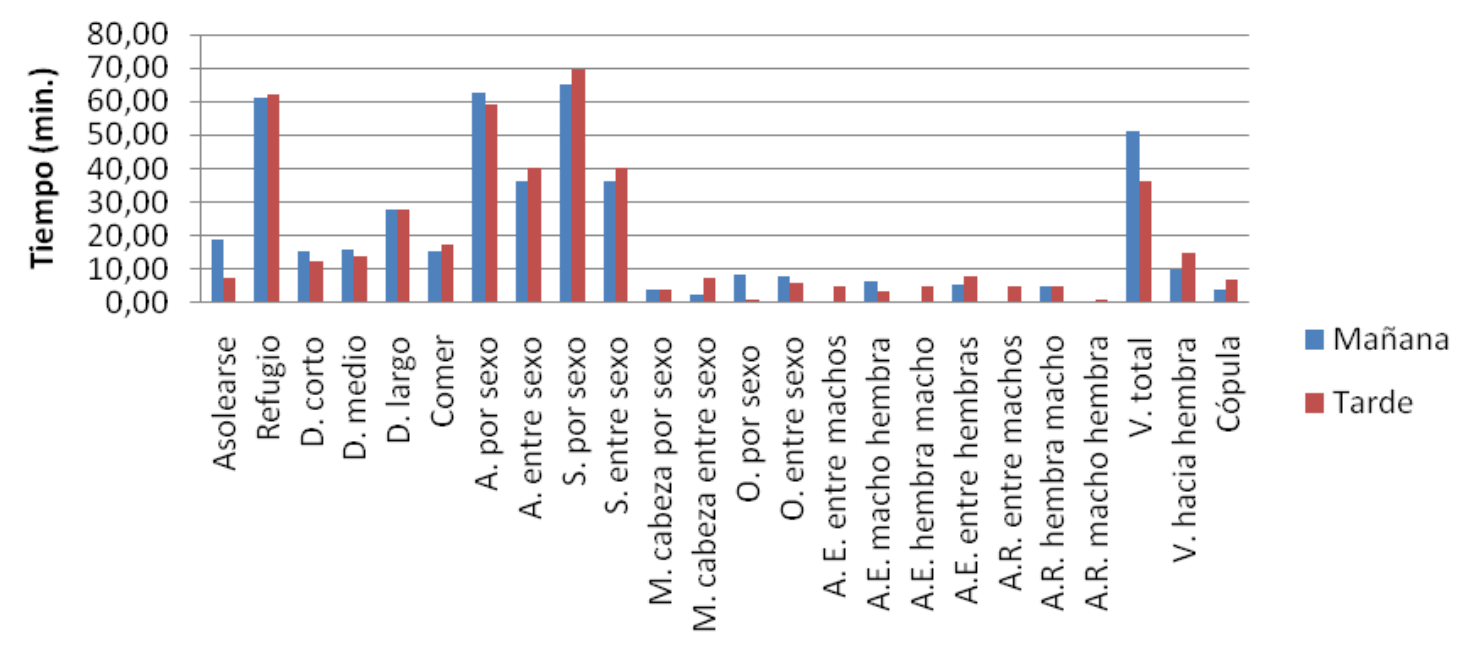

Eventos

Figura 3. Distribución del tiempo empleado por categoría separando entre jornada de la mañana y la jornada de la tarde.

\section{Discusión}

CASTAÑO y LUGO (1979), aportan información referente al comportamiento reproductivo y encontraron que $G$. carbonaria mantenida en cautividad entra en celo precipitadamente por efecto del rápido acostumbramiento al encierro, al iniciar dicho comportamiento, los machos olfatean a las hembras y desisten con facilidad, lo cual es contrarrestado en los días del mes de abril, como se observo en el presente significativamente en el presente estudio.

Aunque la adaptación a las condiciones de cautiverio influye en la reproducción, anticipando la manifestación de eventos propios de este comportamiento, es claro que conservan una tendencia de respuesta al ambiente como lo indica la observación del inicio de la conducta reproductiva a finales de febrero, y el dinamismo aumenta con el inicio de las lluvias, tal como lo señala MOSKOVITS (1988), al afirmar que el cortejo de G. carbonaria es fuertemente sincronizado con el inicio de la estación lluviosa.

Se concuerda con MEDEM et al., (1979), quienes manifiestan que los meses de mayor actividad reproductiva en cautiverio se relacionan con la época de lluvias, pero no se ajustan los datos analizados a lo expuesto por MOSKOVITS (1985), quien manifiesta que $G$. carbonaria es activa reproductivamente después de la temporada de lluvias, lo cual es válido para medio natural, ya que como lo afirma MEDEN (1962), el apareamiento tiene lugar después de la temporada de lluvias, entre julio y septiembre. 
G. carbonaria al igual que Pseudemys floridana y Trachemys scripta, y quelonios en general posee rasgos reproductivos sujetos a la temperatura, precipitación y recursos disponibles en el hábitat (MOLL, 1973; GIBBONS et al., 1981; GIBBONS y GREENE, 1990). Complementariamente se afirma que G. carbonaria durante la sequía prolongada sufre estrés fisiológico, lo que desencadena una atrofia de todo el proceso de formación de los folículos preovulatorios, alterando así la conducta reproductiva a tal punto que cesa la reproducción (GIBBONS et al., 1983).

Los comportamientos descritos en este trabajo se pueden explicar teniendo en cuenta a FITCH (1980), quien plantea que a pesar que las estrategias reproductivas tienen una base genética, también son controladas por las condiciones de manejo y de igual manera por los factores ambientales. Por su parte HERNÁNDEZ y BOEDE (2001), complementan esta afirmación agregando que dicha estrategia es modificada fuertemente por el tamaño del encierro y la densidad de animales; lo que además deja claro que existe reproducción en cautiverio y consecuentemente se manifiestan despliegues reproductivos medibles como los registrados en este estudio.

Por lo visto, los machos dedicaron significativamente parte del tiempo a permanecer en alerta total $(p<0,001)$, ubicándose en lugares estratégicos que les permitieran tener un encuentro con el sexo opuesto, lo cual puede o no presentarse con movimientos de cabeza entre sexos diferentes, así mismo CASTAÑO y LUGO (1979), encontraron en G. carbonaria y G. denticulata una mayor actividad en época de celo en los machos, mientras que en las hembras se dio durante la época de postura. Al parecer el comportamiento percibido en los machos es adoptado como respuesta a la actitud negativa mostrada por las hembras durante las primeras semanas de la temporada reproductiva y puede asociarse como una estrategia que ayudaría a la maduración folicular.

CASARES et al. (1999), plantean una relación endocrinológica para Geochelone nigra, que ayuda a explicar las diferencias de comportamiento reproductivo en función del tiempo y entre sexos: en los machos, la testosterona y corticosterona plasmática aumentan pocos meses antes que comience la estación reproductiva, el pico hormonal se alcanza cuando suceden la mayoría de cópulas; lo que se relacionaría con el inicio temprano de búsqueda de hembras para copular, por su parte en las hembras también se da un incremento de la testosterona y corticosterona pero alcanzan su pico durante la segunda mitad de la estación reproductiva, lo que plantea un lapso diferencial entre ambos procesos y por supuesto diferencias notables de comportamiento, como el de aceptación de la cópula por parte de la hembra, La corticosterona plasmática muestra de manera general estar más elevada en los machos que en las hembras, con variaciones durante el año dependiendo 
del sexo CASARES et al. (1999), y según el comportamiento de apareamiento con diferencias temporales dentro de la estación reproductiva entre sexos.

Durante la toma de datos se registró que $G$. carbonaria en cautiverio tiene claramente definidos lugares destinados al descanso de manera grupal 0 individual, demostrando la habilidad de aprovechar espacios disponibles que brinden confort. Aunque son animales sociales, algunos no toleran la presencia de ciertos individuos en particular. MOSKOVITS (1985) señala el mismo comportamiento en hábitat natural y lo corrobora a través de radio rastreo, encontrando que usualmente descansan largos periodos (3-6 días).

Los machos suelen presentar diferencias en el comportamiento sexual, basándose en la dirección que imprimen en los movimientos de cabeza, vocalización, ángulo de monta y orientación de la cloaca de la hembra, lo cual está de acuerdo con lo señalado por VANZOLINI (1999). Es evidente que los movimientos de cabeza en cautiverio se presentan entre sexos iguales y diferentes, posiblemente se realizan como método de persuasión y/o un mecanismo que denota el estatus jerárquico de los individuos inmiscuidos en una disputa pasiva, según PRITCHARD (1979), ERNEST y BARBOUR (1989), los movimientos facilitan la identificación del sexo opuesto antes de iniciar el ritual de cortejo; LEVEINE y SCHAFER (1992), reportan que los movimientos de cabeza pasan de lado a lado con una serie de sacudidas, al encontrarse con otro morrocoy, sugieren que solo los machos responden a los movimientos. Igual mente, es importante, anotar que G. carbonaria de la misma forma que otros quelonios, utilizan los diseños de línea y bandas coloridas del cuello y miembros, como mecanismos de reconocimiento específicos, por otro lado, las vocalizaciones, señales visuales y olfatorias son utilizadas durante las interacciones sociales (RUEDA et al., 2007).

El comportamiento reproductivo es estacional y la actividad de vocalización y cópula es significativa en las horas de la tarde. El macho persigue a la hembra olfateándola en la zona cloacal, acompañado de cambios comportamentales tales como chocar la parte anterior de su caparazón contra el de la hembra, morder las extremidades y la cola e incluso frotar el rostro de la hembra (BOTERO, 1999), el comportamiento de olfatear decrece en la medida en que avanza la temporada. El desarrollo de este evento permite reconocer durante un encuentro el sexo del individuo e incluso su condición dentro de la jerarquía social.

Novedosamente se describe que el número de sucesos relacionados con los aspectos reproductivos son desarrollados con mayor dinamismo (vocalización, cópula, movimientos de cabeza entre sexo y olfateo entre sexo), mientras que 
el tiempo empleado en cada una de ellas sólo es significativo durante la cópula en las horas de la tarde concordando con AVENDAÑO et al. (2002), además se verifica una baja relación entre cópula efectiva versus encuentros.

La mayoría de las montas no terminan en cópulas, durante la monta los machos cambian la vocalización típica y los choques rítmicos, por jadeos y empujones aislados, al terminar la cópula el macho libera la presión que hace sobre la hembra y ésta a menudo levanta su parte anterior mientras el macho retira el pene (CASTAÑO y LUGO, 1981). Se han registrado durante el cortejo sonidos similares al cacareo generado por las gallinas (SNEDIGAR y ROKOSKY, 1950), el cual puede variar de tono en la medida que se prolonga la cópula, por efecto de abrir y cerrar la boca durante la vocalización, como se observó en el presente trabajo.

El tiempo promedio empleado a la cópula fue de 10,41 (DS=4,5), con un rango de 5 a 25 minutos, lo cual concuerda con MERCADO Y PALACIOS (2003), quienes reportaron un tiempo de 2 a 5 para pequeños ejemplares y 8 a 12 minutos para ejemplares grandes, y con los registros de MERCHAN et al. (1998), quienes hallaron un tiempo de cópula entre 10 y 15 minutos, que se ajusta a la media hallada.

El comportamiento reproductivo puede verse afectado en la medida que el nivel de sociabilidad de los animales disminuye, al aumentar el tamaño del corral o al incrementar el número de refugios disponibles. Los animales tienden a permanecer aislados, limitando la cópula a las hembras más cercanas (AUFFENBERG, 1969). Lo que sin duda podrá ser importante para futuros trabajos comparativos con esta especie a nivel de cautiverio, en donde los resultados aquí obtenidos servirán de base para su discusión.

\section{Referencias}

AUFFENBERG, W. 1969. Social behavior of Geochelone denticulata. Quart. Journ. Florida. Acad. Sci. 32(1):50-58.

AVENDAÑO, I.; MUÑOZ, A.; VARELA, N. 2002. Aproximación al Conocimiento sobre la Reproducción de los Quelonios. Boletín GEAS III(1- 6):42-56

BENNETT, R. A. 1989. Reptil cirugía. Parte I. Principios Básicos. Compendio de Educación Continua para la Práctica de Veterinario 11(1):10-20.

BISBAL, F. 1994. Consumo de fauna silvestre en la zona de Imataca, Estado Bolívar, Venezuela. Interciencia 19(1):28-33.

BOTERO, R. 1994. Comparación de la Incubación Artificial de Huevos de Tortuga Charapa (Podocnemis expansa, Schweigger,1812) Respecto a Huevos 
de Nidos Naturales, en El Río Caquetá, Colombia. Trabajo de Grado. Facultad de Medicina Veterinaria y de Zootecnia. Universidad Nacional de Colombia.

BROWN, C. W.; MARTIN, R. A. 1981. Distocia en Serpientes. Págs. 86-92 en: Johnston, D.E. (ed.), Enfermedades exóticas de los animales en la medicina práctica. Trenton Veterinary Learning Systems.

CASARES, M.; LANCE, V.; SCHRAMM, B. 1999. Steroids levels and reproductive cycle of the Galapagos tortoise, Geochelone nigra, living under seminatural conditions of Santa Cruz island (Galapagos). General and Comparative Endocrinology114:108-120.

CASTAÑO-MORA, O.V.; LUGO, R.M. 1979. Estudio comparativo de dos especies de morrocoy: Geochelone denticulata y Geochelone carbonaria y algunos aspectos de su morfología externa. Trabajo de Grado. Facultad de Ciencias. Departamento de Biología. Universidad Nacional de Colombia. Bogotá, D. E.

CASTAÑO- MORA, O.V.; LUGO, R.M. 1981. Zoocriadero de Morrocoyes, Geochelone carbonaria y Geochelone denticulata. Natura 106 (ID-B).

CASTAÑO-MORA, O.V.; LUGO, R.M.; MEDEM, F. 1979. Contribución al conocimiento sobre la reproducción y el crecimiento de los "Morrocoyes": Geochelone carbonaria y G. denticulata ; Testudines. Testudinidae. Caldasia 12(59):497-511.

CASTAÑO_MORA, O.V. 1992. Informe final del proyecto «Las tortugas de Colombia, fase I/». Universidad Nacional - Colciencias, Bogotá.

CEBALLOS, C. 2000. Tortugas (Testudinata) Marinas y Continentales de Colombia. Biota Colombiana 1(2):187-194.

CITES. 1995. Notificación de las partes № 874. Secretaria de la Convención Sobre el Comercio Internacional de Especies Amenazadas de Fauna y Flora Silvestre. Ginebra. Suiza.

DE LA OSSA, V.J. 1999. Zoocría: Guías académicos introductorias con énfasis en algunos reptiles. Universidad de Sucre, Facultad de Ciencias Agropecuarias, Zootecnia. Sincelejo, Sucre.

KING F.W.; BURKE R.L. 1989. Crocodilian, Tuatara, and Turtle Species of the World .Washington D. C.

ERNST, C.H.; BARBOUR, R.W. 1989. Turtles of the World. Smithsonian Institution Press. Washington D.C.

ESPINOZA, E. 1991. Desarrollo de criaderos de babas Caiman crocodilus, en los llanos venezolanos. II Simposio Nacional Sobre Biotecnología. Universidad del Zulia, Venezuela. Memorias Totales 1:127-135. 
FERRI, V. 1988. El Gran Libro Ilustrado de las Tortugas. Editorial de Vecchi S.A. España.

FITCH, H. 1980. Reproductive strategies of reptiles. Págs. 25-32 en: Murphy B.; Collins, J (eds.), Reproductive biology and disease of captive reptiles. Society for the study of Amphibians and Reptiles. Washington DC.

FREIBERG, M.A. 1971. El mundo de las tortugas. Editorial Albatros. Buenos Aires.

GAFFNEY, E.S. 1984. Historical analysis of theories of Chelonian relationship. Systematic Zoology 33(3):283-301.

GIBBONS, J.W.; SEMLITSCH, R.D.; GREENE, J.L.; SCHUBAUER, J.P. 1981. Variation in age and size at maturity of the Slider Turtle (Pseudemys scripta). American Naturalist 117:841-845.

GIBBONS, J.W.; GREENE, J.L.; CONGDON, J.D. 1983. Drought-related responses of aquatic turtle populations. Journal of Herpetology 17:242-246.

GIBBONS, J.W.; GREENE, J.L. 1990. Reproduction in the slider and other species. Págs. 124-134 en: Gibbons. J.W (ed.), Life History and Ecology of the Slider Turtle. Smithsonian Institution Press. Washington, DC.

GONZÁLEZ, A. 1997. Incubación artificial del Geochelone carbonaria. Facultad de Agronomía, U.C.V. Departamento de Zoología Agrícola. Maracay. Venezuela.

HERNANDEZ, O. 1997. Reproducción y Crecimiento del Morrocoy, Geochelone (Chelonoidis) carbonaria (Spix, 1824), (Testudinidae). Biollania 13:165-183.

HERNÁNDEZ, P.O.; BOEDE, E. 2000. Efecto de la alimentación sobre el crecimiento y producción de huevos de Geochelone (Chelonoidis) carbonaria (spix, 1824) bajo condiciones de cautiverio. Acta Biol. Venez. 20(2):37-43.

HERNÁNDEZ, P.O.; BOEDE, E. 2008. Relación entre el tamaño de hembra y la producción de huevos en el morrocoy sabanero Geochelone (Chelonoidis) carbonaria (spix, 1824) en un zoocriadero comercial de Venezuela. Interciencia 33(6):461-466.

HERNÁNDEZ, C.J y SÁNCHEZ, E. 1992. Biomas terrestres de Colombia. Págs. 105-151 en: La diversidad biológica de Iberoamérica I. Halffter, G (Ed.). CYTED. Inst. Mex. de Ecología y Secretaría de Desarrollo Social. México.

HUTTON, J.; WEBB, G. 1992. Introducción a la Cría de crocodrilianos. Crocodile Specialist Group SSC/IUCN - World Conservation Union. Australia.

IGAC-Instituto Geográfico Agustín Codazzi. 1969. Monografía del departamento de Sucre. Oficina de estudios geográficos. Bogotá DC. 
LEGLER, J.M. 1963. Tortoises (Geochelone carbonaria) in Panama: Distribution and variation. Amer. Midl. Nat. 70(2):490-503.

LEVINE, D.; SCHAFER, D. 1992.Red-footed Tortoise, Geochelone carbonaria. Tortuga Gazette 28(1):1-3.

MADER, D. 1996. Reptile Medicine and Surgery. Saunders W.B. Co. United States of America.

MANZANILLA, J.; PÉFAUR, J. 2000. Consideraciones Sobre Métodos y Técnicas de Campo Para el estudio de Anfibios y Reptiles. Revista Ecológica Latinoamericana 7(1-2):7-30.

MARTIN, P.; BATESON, P. 1991. Measuring Behaviour. An introductory guide Cambridge University Press. Alianza Universidad.

MATIAS, C.; PINHEIRO, F. 2004. La vida silvestre decomisado y rescatado en el estado de Río de Janeiro. Congreso de la Sociedad del Brasil de los Parques Zoológicos. Rio de Janeiro.

MATIAS, R.M.C.A ; PINTO, R.M.A.; TORTELLY, R.; FERREIRE, B.S. 2007. Aspectos fisiopatológicos da retenção de ovos em Jabutipiranga (Geochelone carbonaria Spix, 1824). Ciencia Rural 36(5):1494-1500.

MEDEM, F. 1962. La distribución geográfica y ecología de los Crocodylia y Testudinata en el Departamento del Choco. Revista de la Academia Colombiana de Ciencias Exactas, Físicas y Naturales 11:279-303.

MEDEM, F. 1968. El desarrollo de la herpetología en Colombia. Separata de la Revista de la Academia Colombiana de Ciencias Exactas, Físicas y Naturales 13(50):160-163.

MEDEM, F.D. CASTAÑO, M.; LUGO. M.1979. Contribución al conocimiento sobre la reproducción y el crecimiento de los "morrocoyes" (Geochelone carbonaria y G. denticulata, Testudines, (Testudinidae). Caldasia 12(59):497511.

MERCADO, E.; PALACIOS, R. 2003. Determinación de los patrones más relevantes de comportamiento reproductivo y alimenticio de Geochelone carbonaria (Spix, 1824) bajo condiciones de manejo ex situ. Trabajo de Grado. Facultad de Ciencias Agrpecuarias. Programa de Zootecnia. Universidad de Sucre, Colombia.

MERCHAN, M.; FIDALGO, A.; PÉREZ, C. 1998. Biología, distribución y conservación del morrocoy o tortuga carbonera en los llanos de Venezuela. Reptilia 15:30-38.

MOLL, E.O. 1973. Latitudinal and intersubspecific variation in reproduction of the Painted Turtle, Chrysemys picta. Herpetologica 29:307-318. 
MOSKOVITS, D. 1988. Sexual Dimorphism and Population Estimates of the Two Amazonian Tortoises. (Geochelone carbonaria and G. denticulata) in the Northwestern Brazil. Herpetologica 44(2):209-217.

OJASTI, J. 1993. Utilización de la Fauna Silvestre en América Latina. FAO. Roma, Italia.

PÁEZ, V.P.; RESTREPO, I.A.; BOCK, B.C.; LÓPEZ, O.C. 2006. Efecto de la cosecha sobre la estructura demográfica y genética de dos especies de tortugas continentales. Memorias II Congreso Colombiano de Zoología. Santa Marta, Colombia.

PEREZ, F. 2006. Utilización de cajones para puestas de interior. Una experiencia personal. Disponible en: www.testudinae.com (marzo de 2009).

PIERRET, P.; DOUROJEANNI, M. 1966. La caza y la alimentación humana en las riberas del Río Pachicha, Perú. Turrialba 16(3):271-277.

PORTO, G.R.; PIRATELLI, A. 2005. Etograma de maria-preta, Molothrus bonarensis (Gmelin) (Aves, Emberizidae, Icterinae). Rev. Bras. de Zoología 22(2):306-312.

PRITCHARD, P.C. 1979. Encyclopedia of Turtles. TFH Publications, Neptune, New Jersey. USA.

PRITCHARD, P.C.; TREBBAU, U.P.1984. Turtles of Venezuela. Soc. Study Amphib. Pept. NY. USA.

PRITCHARD, P.C. 1986. Enciclopedia of Turtles. TFH Publications. United States of America.

RUEDA, A.V. 2006. Opciones para la conservación de las tortugas continentales de Colombia: Córdoba, una experiencia de trabajo. $\mathrm{Cl}$ Internacional, CVS. Montería, Córdoba, Colombia.

RUEDA-ALMONACID, J.V.; LYNCH, J.; AMÉZQUITA A. 2004.Libro Rojo de Anfibios de Colombia. Serie Libros Rojos de Especies Amenazadas de Colombia. Conservación Internacional Colombia, Instituto de Ciencias Naturales - Universidad Nacional de Colombia, Ministerio del Medio Ambiente. Bogotá, Colombia. .

RUEDA-ALMONACID, J.V.; CARR, J.L.; MITTERMEIER, R.A.; RIODRÍGUEZMAHECHA, J.V.; MAST, R.B.; VOGT, R.C. RHODIN, A.G.; DE LA OSSA VELÁSQUEZ, J.; RUEDA, J.N.; GOETTSCH MITTERMEIER, C. 2007. Las tortugas y los cocodrilianos de los países andinos del trópico. Conservación Internacional. Bogotá D.C.

RUNDQUIST, E.M. 1994. Breeding the red-footed tortoise in captivity. Captive Breeding 2(3):17-22. 
SNEDIGAR, R.; ROKOSKI, E.J.1950. Courtship and egg laying of captive Testudo denticulata. Copeia 1(1950):46-49.

VANZOLINI, P.E. 1999. A note on the reproduction of Geochelone carbonaria and G. denticulata. (Testudines, testudinidae). Rev. Brasil. Biol. 59(4):593- 608.

ZAR, J.H. 1996. Bioestatistical analysis. Third edition. Prentice-Hall, Inc. Englewood Cliff, N.J.

ZWART, P.; VORSTENBOSCH, C. 1995. Anomalías de la cáscara de los huevos de reptiles. Herpetopathologia. Quinta reunión Internacional de Patología y para reptiles y anfibios. Memorias 1:233-238. 